\title{
Multiphysics Flow Modeling and in Vitro Toxicity of Iron Oxide Nanoparticles Coated with Poly(vinyl alcohol)
}

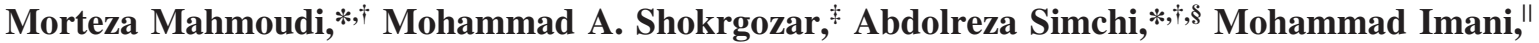 \\ Abbas S. Milani, ${ }^{\perp}$ Pieter Stroeve, ${ }^{\#}$ Hojatollah Vali, ${ }^{\triangleright}$ Urs O. Häfeli, ${ }^{\circ}$ and Shahin Bonakdar \\ Institute for Nanoscience and Nanotechnology and Department of Materials Science and Engineering, Sharif \\ University of Technology, Tehran 11365-8639, Iran, National Cell Bank, Pasteur Institute, Tehran, Iran, \\ Novel Drug Delivery Systems Department, Iran Polymer and Petrochemical Institute, Tehran, Iran, \\ School of Engineering, University of British Columbia Okanagan, Kelowna, Canada, Department of Chemical \\ Engineering and Materials Science, University of California Davis, Davis, California, Department of Anatomy \\ and Cell Biology, McGill University, Montreal, Canada, Faculty of Pharmaceutical Sciences, University of \\ British Columbia, Vancouver, Canada, and Department of Biomedical Engineering, Amirkabir University of \\ Technology, Tehran, Iran
}

Received: October 25, 2008; Revised Manuscript Received: November 7, 2008

This study investigated the behavior of ferrofluids containing superparamagnetic iron oxide nanoparticles (SPION) of various compositions for potential applications in drug delivery and imaging. To ensure biocompatibility, the interaction of these SPION with two cell lines (adhesive and suspended) was also investigated using an MTT (3-(4,5-dimethylthiazol-2-yl)-2,5-diphenyltetrazolium bromide) assay. The cell lines studied were primary mouse connective tissue cells (adhesive) and human leukemia cells (suspended). SPION were synthesized with a co-precipitation method under different stirring rates and $\mathrm{NaOH}$ molarities. The SPION demonstrated a range of magnetic saturations due to their different shapes, which included magnetite colloidal nanocrystal clusters (CNC's), magnetic beads, and single-coated nanoparticles. All synthesized SPION maintained reasonable cell viability following exposure to cells. Flow cytometer tests showed that no apoptosis took place in cells exposed to SPION. A multiphysics numerical model was developed to study the dynamic behavior of ferrofluids containing the SPION in a blood vessel while under an externally applied magnetic field. Simulation results suggest that the SPION magnetic properties and the strength of the external field are important factors in determining both the shape and amplitude of the resulting ferrofluid velocity field.

\section{Introduction}

Due to their ultrafine size and biocompatibility, superparamagnetic iron oxide nanoparticles (SPION) are emerging as promising candidates for biomedical applications, such as in enhanced-resolution magnetic resonance imaging, drug delivery, and cellular targeting. While magnetite and maghemite SPION have been used in biomedical applications, magnetite SPION may be more promising candidates due to better biocompatibility and better magnetic properties. ${ }^{1}$ Their super-paramagnetism is particularly useful in applications such as externally guided drug delivery since removal of the external magnetic field prevents agglomeration and subsequent embolism. ${ }^{1-6}$ However, major shortcomings of these particles in vivo include their destabilization following adsorption of plasma proteins and nonspecific uptake by the reticulum-endothelial system (RES). ${ }^{7,8}$ To

* To whom correspondence should be addressed. Phone: +98-2166164119. Fax: +98-21-66164123. E-mail: mahmoudi@mehr.sharif.edu (M.M.) and simchi@sharif.edu (A.S.).

${ }^{\dagger}$ Institute for Nanoscience and Nanotechnology, Sharif University of Technology.

* Pasteur Institute.

$\S$ Department of Materials Science and Engineering, Sharif University of Technology.

"Iran Polymer and Petrochemical Institute.

${ }^{\perp}$ University of British Columbia Okanagan.

\# University of California Davis.

${ }^{\nabla}$ McGill University.

${ }^{\circ}$ University of British Columbia.

- Amirkabir University of Technology. enhance therapeutic efficacy by inhibiting these phenomena and prolonging circulation times, SPION can be coated with hydrophilic and biocompatible natural or synthetic polymers. ${ }^{9,10}$ This leads to composite heterogeneous particles comprising a magnetic inner core with a modifying outer coating. Due to excellent film forming, emulsifying, and adhesive properties of poly(vinyl alcohol) (PVA), SPION coated with PVA prevent agglomeration. ${ }^{11}$

SPION that evade clearance by the RES and avoid agglomeration, such as PVA-coated magnetite particles, are of particular interest for targeted chemotherapy in cancer patients. Most chemotherapeutic agents possess a narrow therapeutic index, thereby limiting their dosage and resulting efficacy to avoid severe side effects. Noninvasive tumor-targeted chemotherapy using super-paramagnetic, biocompatible, and nanosized delivery vehicles would allow patients to receive increased treatment dosages while minimizing side effects. Potential candidate systems for similar applications have previously been reported. ${ }^{12,13}$

The main objective of this work was to investigate the effect on blood flow of the interaction between an applied magnetic field and the magnetic properties of synthesized SPION. Most recent research investigating SPION is dedicated to the early detection of cancer, diabetes, and atherosclerosis. This paper focuses on early detection of atherosclerosis in major arteries (vessel size and speed data given by Saltzman ${ }^{14}$ ). The ability to detect arterial plaque formation 


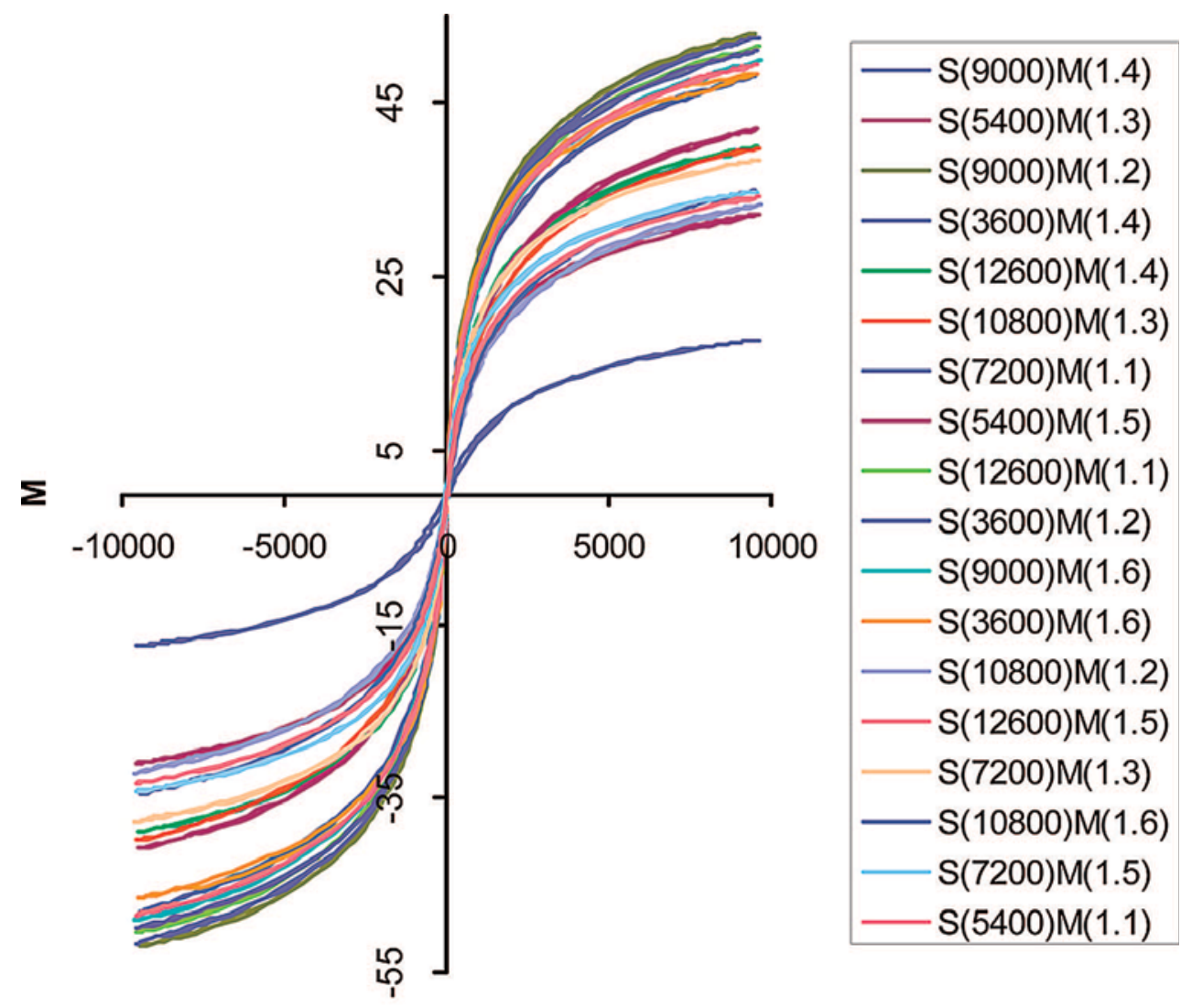

H

Figure 1. VSM curves for the 18 synthesized samples (H, applied magnetic field (Oe); M, magnetization (emu/g)). ${ }^{16}$ The SPION sample code is $\mathrm{S}(x) \mathrm{M}(x)$, where $S$ is the stirring rate and $M$ is the $\mathrm{NaOH}$ molarity

during the early stages of development would identify potential causes of blood flow restriction, and thereby ultimately prevent myocardial infarction. The biocompatibility of prepared SPION was initially assessed using in vitro assays. Since the behavior of magnetic particles in a viscous medium can be modeled using the finite element method (FEM) ${ }^{15}$ a numerical analysis framework was then employed to simulate how these particles would function in vivo when subjected to applied fields of various strength. Although information about the toxicity of SPION continues to increase, a significant knowledge gap still exists on a complete toxicological profile of these promising magnetic materials proposed for future use in many aspects of biomedical engineering. Without the data, risk assessment or regulation for safety of the materials shall suffer immeasurably. To fill the information gap, we also conducted a study to assess the toxic effect of SPION on the cellular viability of L929 (adhesive) cells in vitro.

\section{Materials and Methods}

2.1. SPION Biocompatibility Assessment. 2.1.1. Particle Preparation. Poly(vinyl alcohol) (PVA; $M_{\mathrm{W}}=30000-40000$; degree of hydrolysis, 86-89\%) was purchased from Fluka (Germany). Analytical grade ferrous and ferric chloride $\left(\mathrm{FeCl}_{2}\right.$ and $\left.\mathrm{FeCl}_{3}\right)$ and sodium hydroxide $(\mathrm{NaOH})$ were purchased from Merck (Darmstadt, Germany) and used without further processing.

Solutions were prepared by bubbling argon through deionized (DI) water for $30 \mathrm{~min}$ for deaeration. Iron salts were dissolved in DI water containing $0.5 \mathrm{M} \mathrm{HCl}$, with the mole fractions of $\mathrm{Fe}^{2+}$ to $\mathrm{Fe}^{3+}$ adjusted to 2:1 for all samples. Particle precipitation was performed by dropwise addition of iron salt solutions to $\mathrm{NaOH}$ solutions under an argon atmosphere. To prevent the development of large polycrystalline particles, turbulence was created by placing the reaction flask in an ultrasonic bath and controlling the homogenization rate $(3600-9000 \mathrm{rpm})$ during the initial 2 min of reaction; various $\mathrm{NaOH}$ molarities where also examined (see Mahmoudi et al. ${ }^{16}$ ). After $30 \mathrm{~min}$, PVA solution (polymer to iron mass ratio of 2) was added as a stabilizer, and the reaction proceeded $\left(3600 \mathrm{rpm}, 35^{\circ} \mathrm{C}\right)$ for an additional $30 \mathrm{~min}$. PVA-coated SPION were subsequently collected by centrifugation at $2000 \mathrm{~g}$ for $10 \mathrm{~min}$ and re-dispersed in DI water. The resulting ferrofluid was stored at $4{ }^{\circ} \mathrm{C}$ for future use.

To recall the synthesis parameters of a particular sample, samples are herein referred to as $\mathrm{S}(x) \mathrm{M}(x)$, where $S$ is the stirring rate and $M$ is the $\mathrm{NaOH}$ molarity. For instance, $\mathrm{S}(3600) \mathrm{M}(1.2)$ indicates a sample prepared with a stirring rate of $3600 \mathrm{rpm}$ and a molarity of 1.2. Complete information regarding synthesis, characterization methods (X-ray diffraction (XRD), Fourier transform infrared (FT-IR), UV/vis, transmission electron microscopy (TEM), thermogravimetric analysis (TGA), and differential thermal analysis (DTA)), and particle core and hydrodynamic sizes are reported in a previous study. ${ }^{16}$

2.1.2. In Vitro Biocompatibility Assessment. Since SPION are thought to have a shelf life of 6 months, cytotoxicity was studied using 6 month old samples. Fresh samples were not studied since reports are available in the literature documenting the biocompatibility of freshly prepared SPION. ${ }^{17-19}$ 


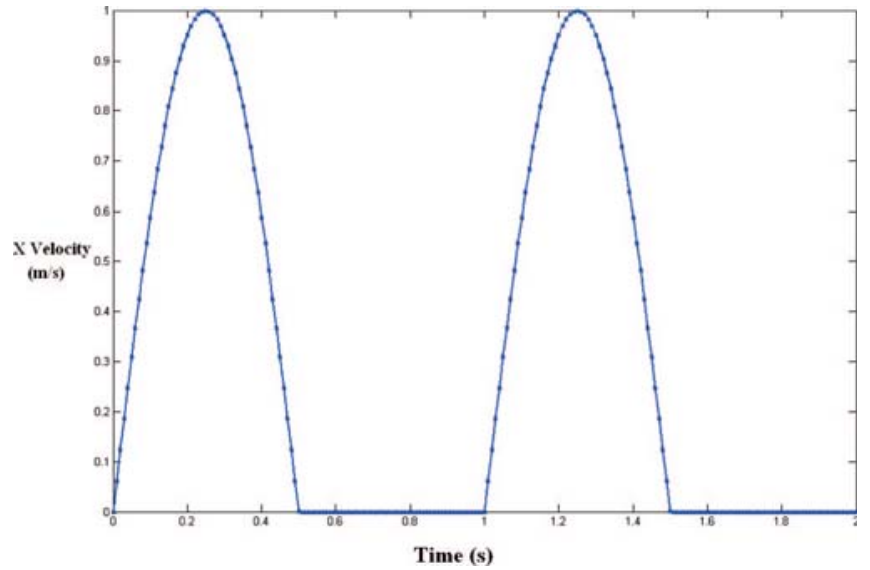

Figure 2. $X$ velocity of blood at the input boundary of the vessel. The plotted function is based on a heart beat model of 60 beats/min.

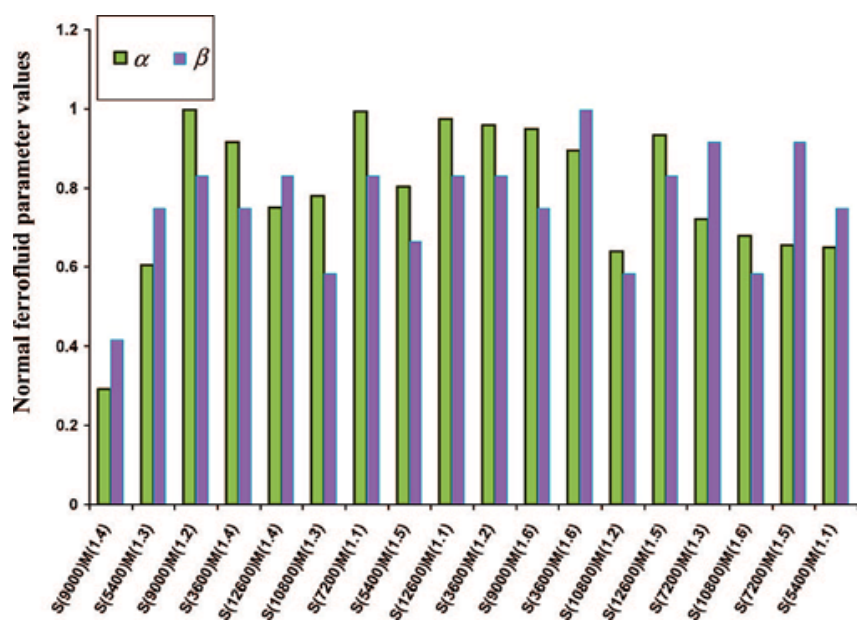

Figure 3. Normal ferrofluid parameters, $\alpha$ and $\beta$, estimated for the 18 synthesized samples. Note that these parameters represent, respectively, the saturation magnetization and the initial susceptibility of ferrofluids to magnetization. The SPION sample code is given in Figure 1 .

Primary mouse fibroblasts (L929, adhesive) and human leukemia cells (K562, suspended) from the National Cell Bank of Iran (NCBI), Pasteur Institute, were seeded on glass coverslips in 96-well plates at 10000 cells/well in $150 \mu \mathrm{L}$ of medium and incubated for $24 \mathrm{~h}$. Cells were cultured in Dulbecco's modified Eagle's medium (DMEM) supplemented with $10 \%$ fetal bovine serum (FBS) at $37{ }^{\circ} \mathrm{C}$ in a $5 \% \mathrm{CO}_{2}$ incubator. After the $24 \mathrm{~h}$ incubation period, medium containing SPION $(0.2,1,5$, and $20 \mathrm{mM}$ iron, measured by atomic absorption) was added to the wells, and cells were incubated for additional periods ranging from 3 to $48 \mathrm{~h}$. Control cells were incubated with the same culture medium without particles. All particle concentrations and controls were each seeded in five separate wells.

Cytotoxicity was assessed using the MTT (3-(4,5-dimethylthiazol-2-yl)-2,5-diphenyltetrazolium bromide) assay, which is a nonradioactive, colorimetric assay. ${ }^{19}$ After 3, 24, and $48 \mathrm{~h}$ of incubation of the cells with the SPION samples, $100 \mu \mathrm{L}$ of MTT $(0.5 \mathrm{mg} / \mathrm{mL})$ was added to each well. Following incubation, the medium was removed and formazan crystals were solubilized by incubation for $20 \mathrm{~min}$ in $150 \mu \mathrm{L}$ of 2-propanol. The absorbance of each well, which assesses viable cells, was
TABLE 1: Fluid (Blood) Physical Constants

\begin{tabular}{ll}
\hline fluid physical constants & value \\
\hline density $(\rho)$ & $1000 \mathrm{~kg} / \mathrm{m}^{3}$ \\
dynamic viscosity $(\eta)$ & $5 \mathrm{e}^{-3} \mathrm{NCs} / \mathrm{m}^{2}$
\end{tabular}

TABLE 2: Magnetic Constants of the Nanoparticles

\begin{tabular}{ll}
\hline \multicolumn{1}{c}{ magnetic constants } & \multicolumn{1}{c}{ value } \\
\hline ferrofluid parameter $(\alpha)$ & values taken from Figure 1 \\
ferrofluid parameter $(\beta)$ & values taken from Figure 1 \\
magnet magnetization & $0.5 \mathrm{e}^{5}$ \\
$\mu_{\mathrm{r}}$ & $5 \mathrm{e}^{3}$ \\
tissue relative permeability & 0.9998
\end{tabular}

read at $545 \mathrm{~nm}$ on a microplate reader (Stat Fax-2100, AWARENESS, Palm City, FL).

2.1.3. Outlier Detection. All MTT experiments were performed in triplicate or more, with the results expressed as mean \pm standard deviation; standard deviation values are indicated as error bars in the MTT results plots. The results were statistically processed for outlier detection using a "T procedure" ${ }^{20}$ using MINITAB software (Minitab Inc., State College, PA). One-way analysis of variance (ANOVA) with $p<0.05$ was performed for each set of MTT assay test repeats. Outlier samples have then been excluded from the corresponding asset viability calculations.

2.1.4. Apoptosis Measurement. An ubiquitous feature of apoptosis is the breakup of chromatin, resulting in the exposure of numerous $3^{\prime} \mathrm{OH}$ DNA ends. When the DNA of cells undergoing apoptosis is analyzed by gel electrophoresis, a distinctive ladderlike appearance of DNA pieces with discrete molecular weights is observed. A quick way to assess apoptosis is then to compare the mobility of DNA extracted from nonapoptotic cells to that of cells which have been induced to undergo apoptosis, such as comparing DNA mobility of untreated Jurkat cells to the mobility of DNA of camptothecininduced Jurkat cells. ${ }^{21,22}$ To determine cellular apoptosis due to the exposure of SPION, the Apoptosis APO-BRDU ${ }^{\mathrm{TM}}$ kit by dual color flow cytometry and microscopy was used (Invitrogen Corp., Carlsbad, CA).

The kit provides an easy method of assessing apoptosis; however, the use of this kit requires that the cells under study are first lysed. The appearance of the $3^{\prime} \mathrm{OH}$ ends can also be quantified as a measure of apoptosis in whole cells by an alternative method which does not require cell lysis. Such an alternative method for use in mixed-cell populations is called the TUNEL assay (terminal deoxynucleotidyl transferase-mediated dUTP nick end-labeling), also known as the bromodeoxyuridine terminal deoxynucleotidyl transferase assay. L929 cells $\left(3 \times 10^{6}\right)$ were placed in a flask and PVAcoated SPION $(200 \mathrm{mM} / \mathrm{mL})$ added for $72 \mathrm{~h}$. Identical cultures without added SPION were used as controls. Cells were then fixed with paraformaldehyde in phosphate-buffered saline (PBS), followed by ethanol fixation. The cells were then washed and reacted with the TdT enzyme (terminal deoxynucleotidyl transferase) and Br-dUTP (bromodeoxyuridine triphosphate) in buffered solution at $37{ }^{\circ} \mathrm{C}$ for $60 \mathrm{~min}$. Bromodeoxyuridine was covalently incorporated into the $3^{\prime}$ DNA ends during this incubation. Cells were then thoroughly rinsed and incubated with a FITC (fluorescein isothiocyanate) labeled antibody directed to bromodeoxyuridine for $30 \mathrm{~min}$. After washing away unbound antibody, immunostaining with 


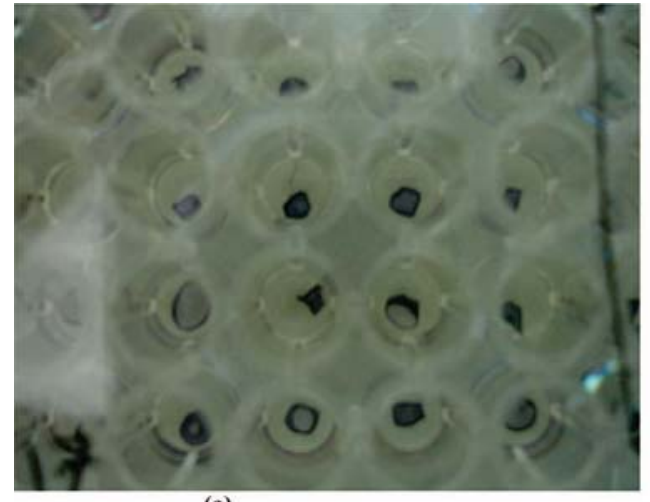

(a)

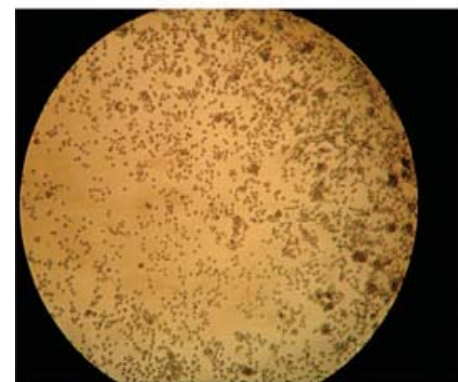

(C)

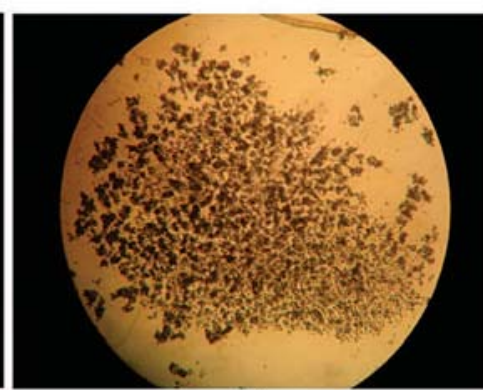

(d)

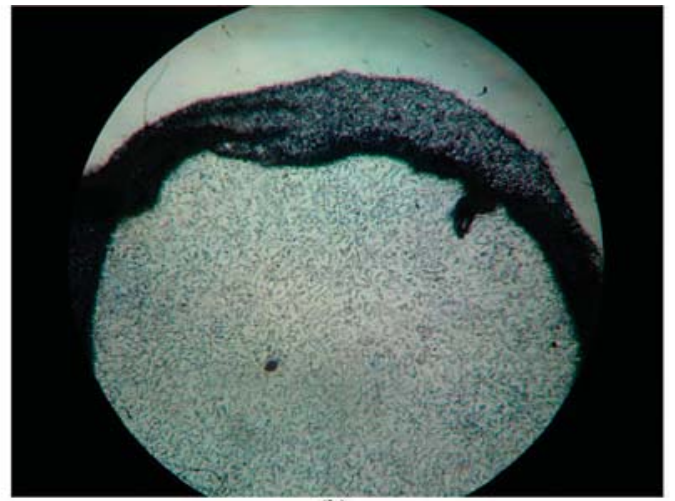

(b)

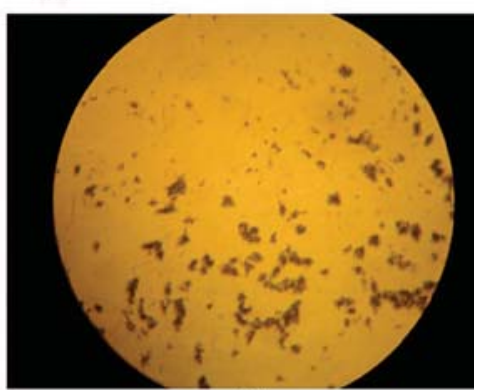

(e)

Figure 4. (a) Photograph and (b) optical microscopy of colonies (L929) in wells containing SPION after treatment with MTT for 4 h; (c) optical MTT for suitable treatment time $(\sim 2 \mathrm{~h})$; ( $\mathrm{d}$ and e) optical microscopy for $4 \mathrm{~h}$ and suitable time, respectively.

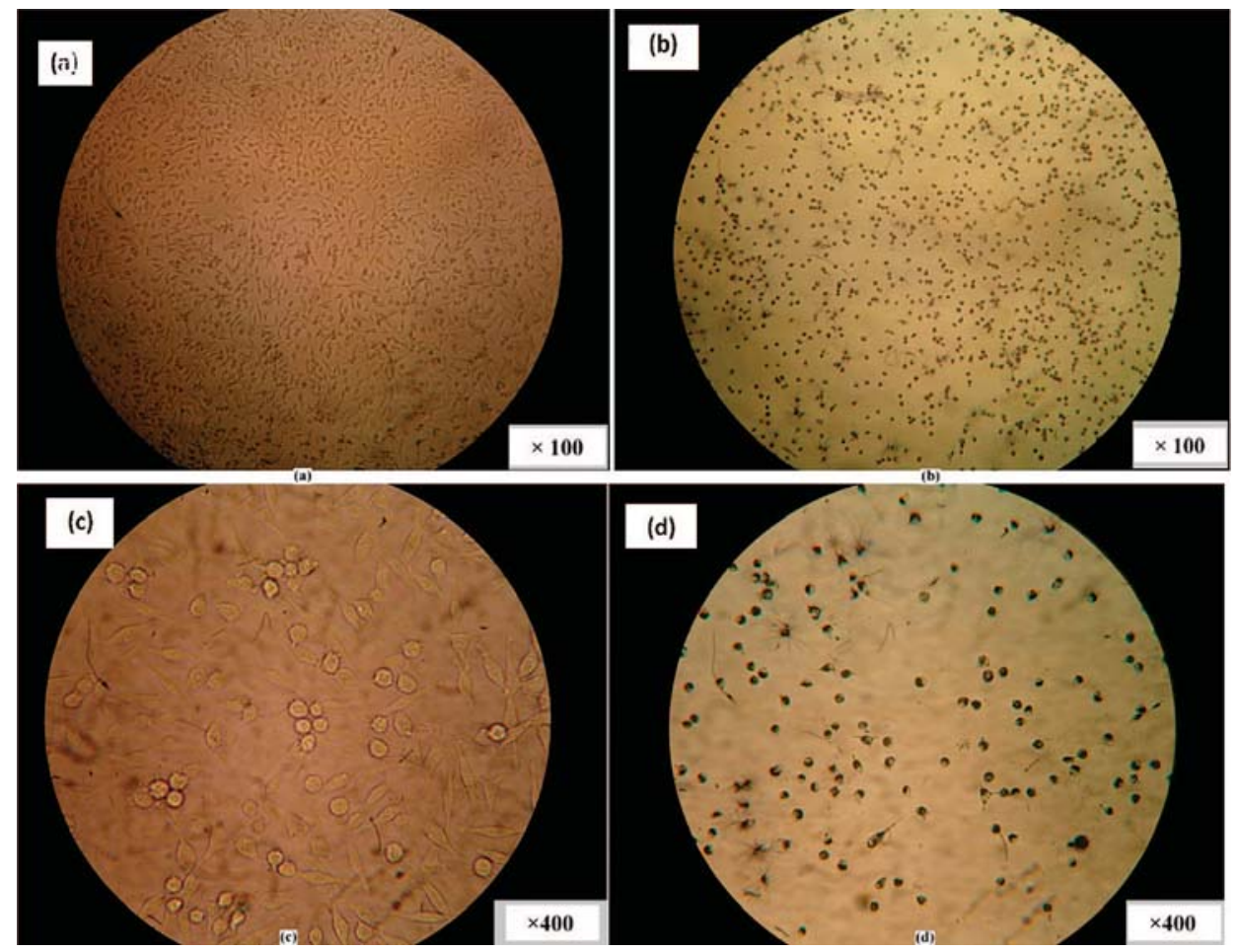

Figure 5. Optical microscopy of L929 cells containing SPION before (a and c) and after (b and d) MTT treatment.

the FITC labeled antibromodeoxyuridine antibody was indicative of the number of free $3^{\prime}$ ends. The RNA of the cells was then digested and the total DNA stained by incubation with a solution containing RNase A plus propidium iodide. Staining of cells with propidium iodide allows normalizing FITC staining to the total amount of DNA in the cells. The stained cells were then analyzed by flow cytometry (FACScan Becton Dickinson, Mountain View, CA) with an argon laser emitting at $488 \mathrm{~nm}$. FITC fluorescence was observed at $520 \mathrm{~nm}$ and propidium iodide simultaneously at $623 \mathrm{~nm}$.

2.2. Numerical Modeling of Nanoparticles Flow in an Applied Field. To investigate how different magnetic properties and particle size affect blood flow conditions, the flow dynamics of ferrofluid containing SPION under an applied static magnetic field was studied by iteratively solving coupled Maxwell and 

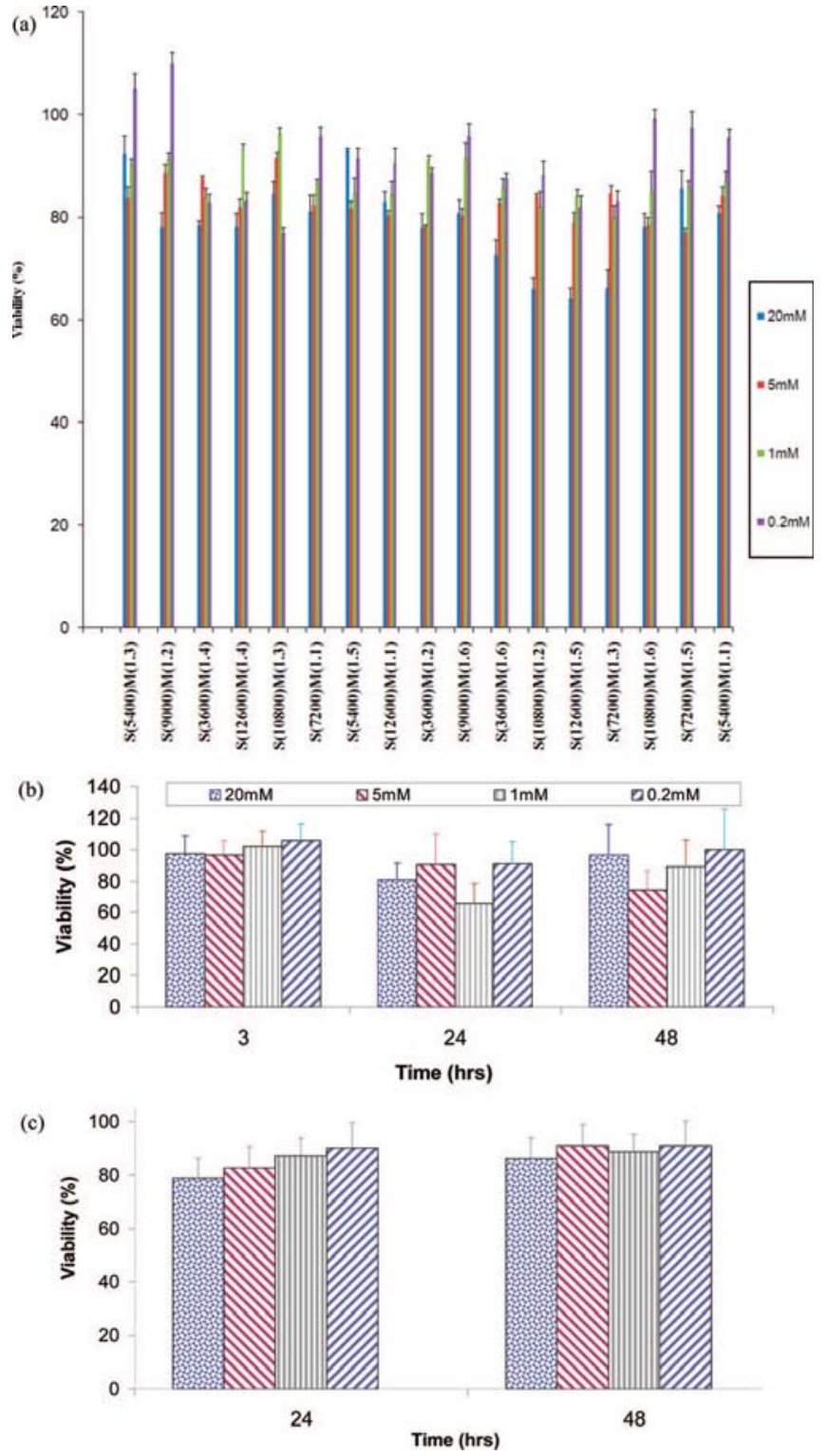

Figure 6. (a) Cell viability of MTT assay results for all SPION samples on K562 cells over $24 \mathrm{~h}$. Average MTT assay values for L929 (b) and K562 (c) cells. The control standard deviations (SD) were 3.43 and 2.31 , respectively. Concentrations are for the amount of SPION. The SPION sample code is given in Figure 1.

Navier-Stokes equations via a finite element approach in COMSOL (COMSOL Inc., Burlington, MA). More specifically, a multiphysics numerical model was developed to study the effects of (a) interactions between magnetic properties of the SPION and (b) the strength of the applied field, on the amplitude and shape of the resulting ferrofluid velocity field. The main governing equations and physical constants of this model are briefly described.

2.2.1. Maxwell's Equations. The static case for Maxwell's equation is defined by ${ }^{23}$

$$
\begin{gathered}
\nabla \times \mathbf{H}=\mathbf{J} \\
\nabla \cdot \mathbf{B}=0 \\
\mathbf{B}=\mu_{0} \mu_{\mathrm{r}}(\mathbf{H}+\mathbf{M})
\end{gathered}
$$

where $\mu$ is the permeability $\left(\mu=\mu_{0} \mu_{\mathrm{r}}\right), \mathbf{B}$ is the magnetic induction, $\mathbf{H}$ is magnetic field strength, $\mathbf{M}$ is the magnetization vector, and $\mathbf{J}$ is the induced current density. It is worth noting
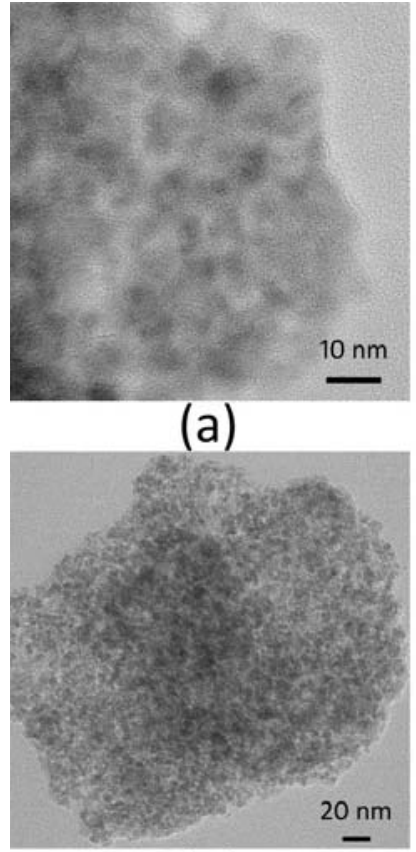

(c)

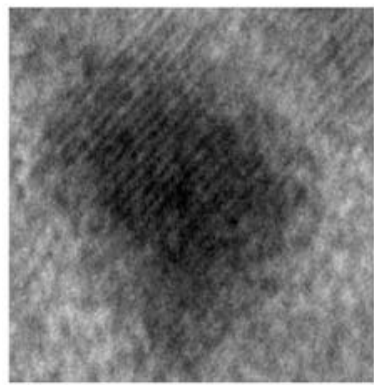

(e) (a)

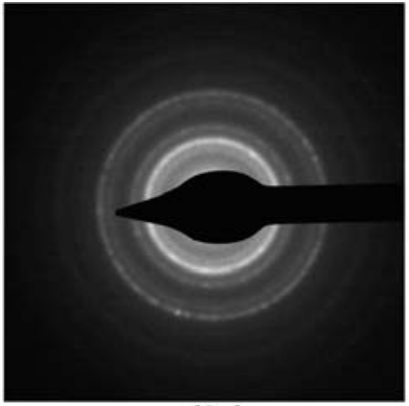

(b)

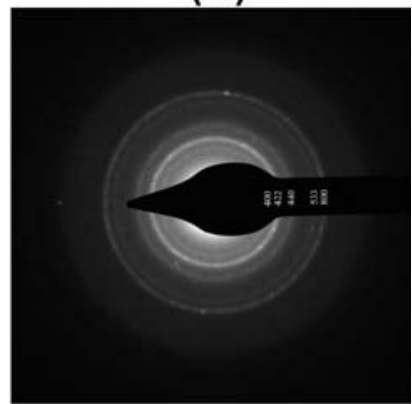

(d)

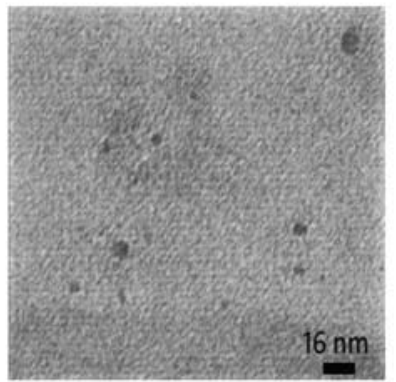

(f)
Figure 7. (a and c) TEM images of nanosized SPION with PVA polymeric beads ( $\mathrm{S}(10800) \mathrm{M}(1.3)$ ). (b and d) TEM diffraction patterns SPION showing the magnetite phase (spinel structure) for a and c, respectively. (e) Magnetite lattice fringes. (f) Single SPION without beads.

that $\mathbf{B}=\nabla \times \mathbf{A}$ and $\nabla \cdot \mathbf{A}=0$, where $\mathbf{A}$ is the magnetic vector potential and $\nabla$ is the gradient operator. The following equations can then be derived:

$$
\begin{gathered}
\nabla \times \mathbf{A}=\mu(\mathbf{H}+\mathbf{M}) \\
\nabla \times\left(\mu^{-1} \nabla \times \mathbf{A}\right)=\nabla \times \mathbf{H}+\nabla \times \mathbf{M} \\
\nabla \times\left(\mu^{-1} \nabla \times \mathbf{A}-\mathbf{M}\right)=\mathbf{J}
\end{gathered}
$$

Since this work considers a $2 \mathrm{D}$ model, eq 6 can be rewritten $\operatorname{as}^{23}$

$$
-\nabla \cdot\left(\mu^{-1} \nabla A-\gamma\right)=J
$$

where $\gamma$ replaces $\mathbf{M}$, magnetization of the ferrofluid, and is approximated $\mathrm{as}^{24}$

$$
\gamma=\left(\alpha \arctan \left(\beta \frac{\partial A(x, y)}{\partial x}\right), \quad \alpha \arctan \left(-\beta \frac{\partial A(x, y)}{\partial y}\right)\right)
$$

Note that, for a 2D model, $A=A_{z}$ and $J=J_{z} . \alpha$ and $\beta$ are model constants computed by fitting the arctangent function of eq 8 to the vector space model (VSM) curves obtained for the SPION (Figure 1).

2.2.2. Navier-Stokes Equations. The dynamic representation of ferrofluid motion is based on a momentum conserva- 
(a)
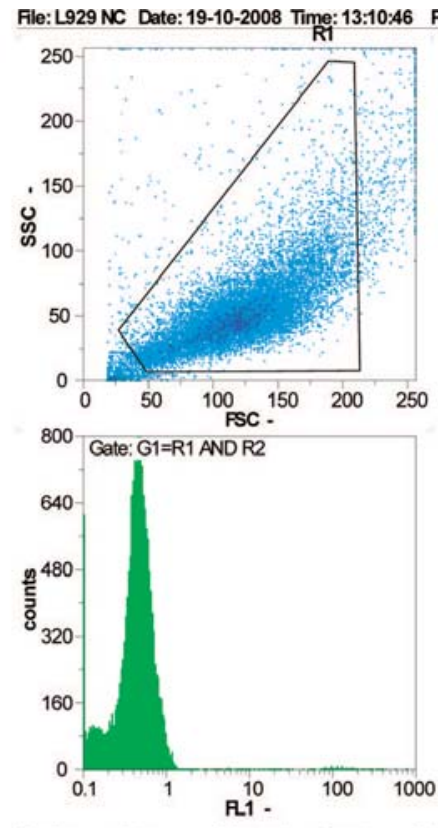
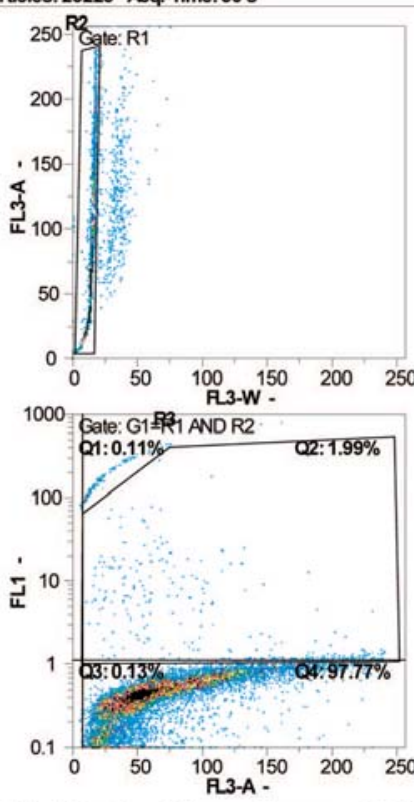

Count/ml \%Gated GM-x Mean-x CV-x\%

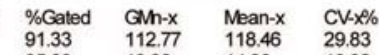
$\begin{array}{llll}91.33 & 112.77 & 118.46 & 29.83 \\ 9560 & 13.83 & 14.00 & 13.92\end{array}$ $\begin{array}{llll}95.60 & 13.83 & 14.00 & 13.92 \\ 0.11 & 6.81 & 6.83 & 7.37\end{array}$ $\begin{array}{llll}0.11 & 6.81 & 6.83 & 7.37 \\ 1.99 & 50.65 & 79.65 & 90.46\end{array}$ $\begin{array}{llll}1.99 & 50.65 & 79.65 & 90.46 \\ 0.13 & 6.94 & 6.97 & 9.46\end{array}$ $\begin{array}{llll}0.13 & 6.94 & 6.97 & 9.46 \\ 97.77 & 55.19 & 63.59 & 55.71\end{array}$ $\begin{array}{llll}97.77 & 55.19 & 63.59 & 55.71 \\ 1.66 & 101.10 & 127.82 & 57.09\end{array}$
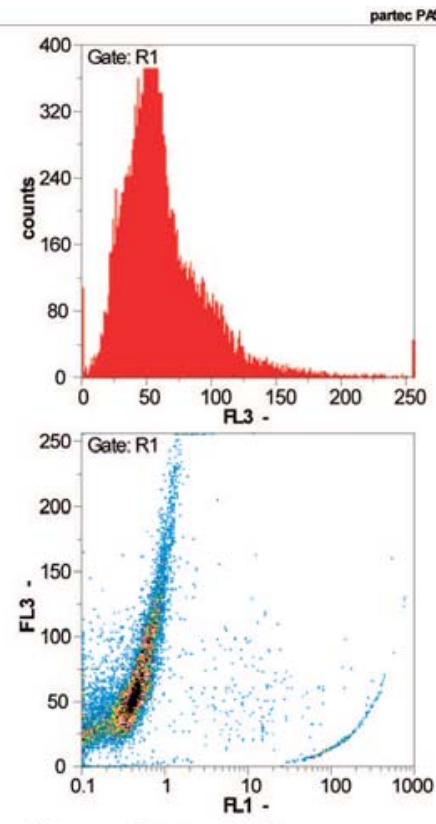

GMn-y Mean-y CV-y\%

$\begin{array}{lll}45.94 & 50.74 & 47.09 \\ 54.82 & 63.78 & 57.46\end{array}$

$\begin{array}{lll}54.82 & 63.78 & 57.46 \\ 76.52 & 78.71 & 17.05\end{array}$

$\begin{array}{lll}76.52 & 78.71 & 17.05 \\ 17.22 & 81.39 & 148.31\end{array}$

$\begin{array}{lll}17.22 & 81.39 & 148.31\end{array}$

$\begin{array}{lll}0.12 & 0.13 & 41.77 \\ 0.40 & 0.45 & 43.66\end{array}$

$\begin{array}{lll}0.40 & 0.45 & 43.66 \\ 3.07 & 9.36 & 320.24\end{array}$ (b)
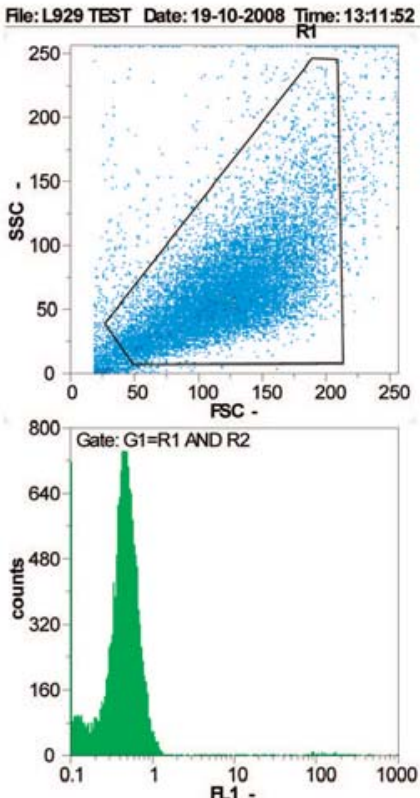

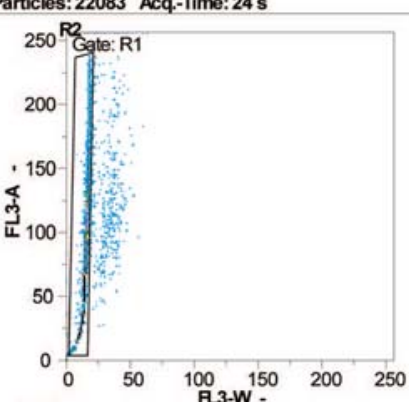

1000 Gate: G1 RR1 AND R2

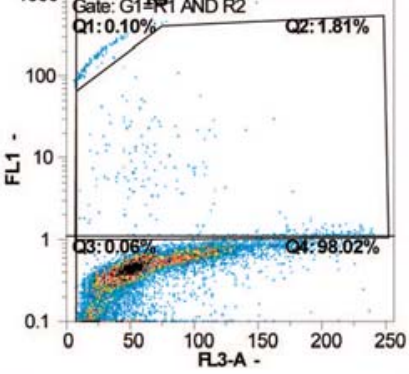

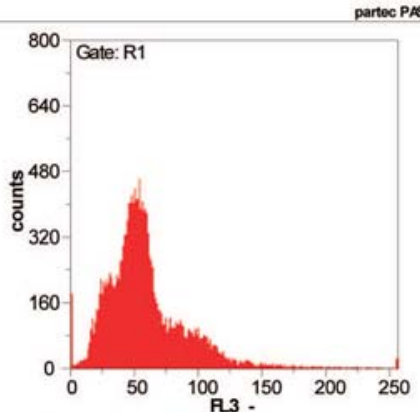

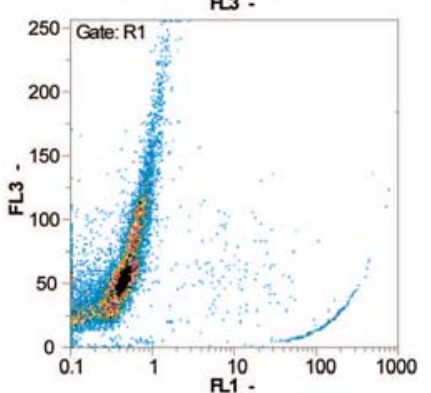

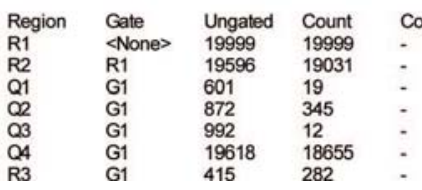

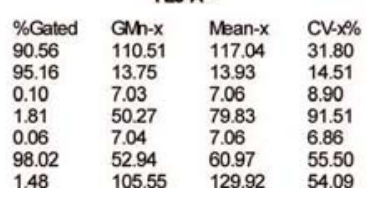

GMn-y Mean-y CV-y\%

$\begin{array}{lll}53.68 & 60.36 & 49.62\end{array}$

$\begin{array}{lll}52.72 & 61.23 & 57.29\end{array}$

$\begin{array}{lll}83.36 & 83.52 & 6.28\end{array}$

$\begin{array}{lll}18.38 & 80.86 & 142.05\end{array}$

$\begin{array}{lll}0.12 & 0.12 & 37.56\end{array}$

$\begin{array}{lll}0.40 & 0.45 & 43.38 \\ 2.98 & 9.40 & 353.90\end{array}$

Figure 8. Flow cytometery results for L929 Cells (a) with no SPION added and (b) with $200 \mathrm{mM}$ SPION added.

tion equation that assumes an incompressible Newtonian flow, as given by the Navier-Stokes equations. ${ }^{25}$ The general vector form of these equations for an incompressible flow is given by eq 9 . Equation 10 is the continuity of mass, where $D$ is the substantive derivative operator. In eqs 9 and $10, \rho$ is the density, $\eta$ is the viscosity, $t$ is time, $\mathbf{u}$ is the velocity vector, and $p$ denotes the fluid pressure. $\nabla \mathbf{u}$ is the tensor derivative of the velocity vector denoting convective acceleration. Conventionally, for a nonrotational fluid $(\nabla \times \mathbf{u}$ $=\mathbf{0})$, the latter derivative is often written as $(\mathbf{u} \cdot \nabla) \mathbf{u}$. The pressure effect is represented by $\nabla \rho$, and the viscosity contribution is $\eta \nabla^{2} \mathbf{u}$. The external force vector $\mathbf{F}=\left(F_{x}, F_{y}\right)$ includes the magnetic volume force and gravity $\left(F_{j}=F_{j}^{\text {mag }}\right.$ $+F_{j}^{\text {grav }} ; j=x$ or $y$ ). The magnetic force components for a 2D field are described by eqs 11 and $12 .^{26}$

$$
\begin{gathered}
\rho \frac{\partial \mathbf{u}}{\partial t}-\eta \nabla^{2} \mathbf{u}+\rho(\mathbf{u} \cdot \nabla \mathbf{u})+\nabla p=\mathbf{F} \\
\frac{\mathrm{D} \rho}{\mathrm{D} t}+\rho(\nabla \cdot \mathbf{u})=0
\end{gathered}
$$




$$
\begin{aligned}
& F_{i}^{\mathrm{mag}}(x, y)=\mu_{0}\left(M_{i}(x, y)+M_{j}(x, y)\right)^{1 / 2} \mu^{-1} \times \\
& {\left[\left(\frac{\partial A(x, y)}{\partial x} \frac{\partial^{2} A(x, y)}{\partial x \partial x}+\frac{\partial A(x, y)}{\partial y} \frac{\partial^{2} A(x, y)}{\partial x \partial y}\right) /\right.} \\
&\left.\left(\left(\frac{\partial A(x, y)}{\partial y}\right)^{2}+\left(\frac{\partial A(x, y)}{\partial x}\right)^{2}\right)^{1 / 2}\right] \\
& F_{j}^{\mathrm{mag}}(x, y)=\mu_{0}\left(M_{i}(x, y)+M_{j}(x, y)\right)^{1 / 2} \mu^{-1} \times \\
& {\left[\left(\frac{\partial A(x, y)}{\partial x} \frac{\partial^{2} A(x, y)}{\partial y}+\frac{\partial A(x, y)}{\partial y} \frac{\partial^{2} A(x, y)}{\partial y \partial y}\right) /\right.} \\
&\left.\left(\left(\frac{\partial A(x, y)}{\partial y}\right)^{2}+\left(\frac{\partial A(x, y)}{\partial x}\right)^{2}\right)^{1 / 2}\right]
\end{aligned}
$$

For boundary conditions, a nonslip wall condition was applied to the vessel wall; i.e., $\mathbf{u}=(0,0)$. At the vessel inlet, we applied a parabolic $x$-velocity flow profile in the form of " $\left(v_{0} / 4\right) S(1-$ $S) \operatorname{amp}[\sin (\omega t)+\sin (\omega t)]$ ". $S$ is a model parameter which varies between 0 and 1 . We assumed a heart rate of 60 beats $/ \mathrm{min}(\omega$ $=2 \pi \mathrm{rad} / \mathrm{s}$, sinusoidal) and a peak velocity of $v_{0}=1 \mathrm{~m} / \mathrm{s}$ for all simulations. A typical input flow profile is depicted in Figure 2.

To avoid numerical instabilities inherent in coupling the differential equations (i.e., Maxwell and Navier-Stokes equations), as well as singularities that may occur in nonlinear finite element methods (FEM), we used an adaptive meshing scheme in COMSOL to facilitate smoothness of the solution. Adaptive meshing in FEM permits mesh refinements in regions that require higher numerical resolutions. In addition to optimum memory performance, adaptive meshing also normally requires fewer convergence iterations.

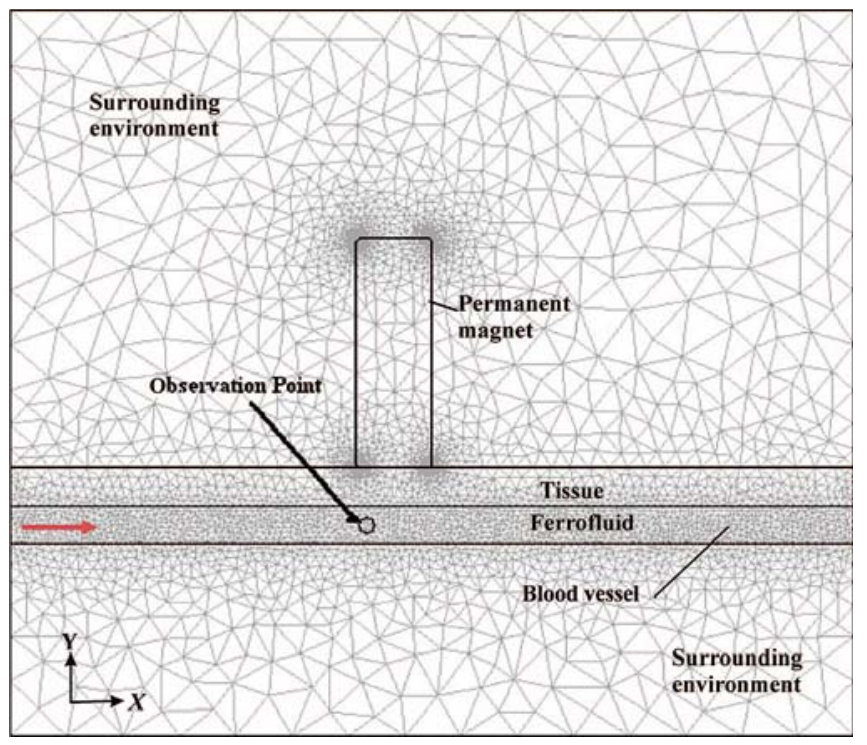

Figure 9. Two-dimensional geometry and finite element mesh model of a blood vessel under a permanent (static) magnetic field for drug delivery.

TABLE 3: Boundary Condition (BC) of the Static Magnetic Field and Fluid Flow

\begin{tabular}{lll}
\hline \multicolumn{1}{c}{ magnetostatic } & fluid & \multicolumn{1}{c}{$\mathrm{BC}$} \\
\hline magnetic insulation & & $A_{Z}=0$ \\
constitutive relation & & $B_{0}=\mu_{0} \mu_{\mathrm{r}} H+B_{\mathrm{r}}$ \\
relative permeability & & isotropic \\
& inflow & $u=$ heart beat equation \\
& outflow & $p_{0}=0$ \\
& wall & $u=0$
\end{tabular}

2.2.3. Physical Constants. The physical constants used to model blood are shown in Table 1. Table 2 includes the magnetic parameters used for Maxwell's equations. Figure 3 provides the normalized $\alpha$ and $\beta$ values of ferrofluid constants for the various synthesized SPION. The key $\alpha$ and $\beta$ parameters represent the saturation magnetization and the initial susceptibility of ferrofluids to magnetization. To this end, the magnetization function of eq 8 was curve-fitted to the VSM results for SPION (Figure 1). ${ }^{3,16}$ It was assumed that the error variance under testing was normal and did not vary with the level, $\mathbf{H}$, of the applied magnetic field. ${ }^{27}$

\section{Results and Discussion}

3.1. MTT Results. 3.1.1. Biocompatibility of SPION-MTT Assay. MTT reduction was used to metabolically quantify viable cells after exposure to SPION. Since published reports confirm that use of the MTT assay for measuring the toxicity of magnetite nanoparticles has high variability and nonspecificity, ${ }^{28}$ the outlier detection method was applied to minimize variability. Cell detachment upon exposure to SPION also necessitated the development of customized protocols for the MTT assay; detachment increased with both increasing SPION concentration and contact time. During the assay, cells exposed to SPION detached from the wells after $4 \mathrm{~h}$ (Figure 4). In reducing the adhesive properties of L929 cells, SPION exposure may have increased error in the MTT assay through the elimination of crystals during removal of the supernatant.

To accommodate for cell detachment, cells were examined by optical microscopy to ascertain the density of violet spots prior to detachment (Figure $4 c-e$ ). The supernatant was then carefully removed to quantify precipitated formazan. For samples exposed to SPION concentrations of $20 \mathrm{mM}$ over $48 \mathrm{~h}$, cell detachment occurred prior to MTT addition. This required a reduction in the MTT incubation time to about $2 \mathrm{~h}$ to avoid cell detachment (Figure 5). The MTT protocol customization also included the removal of iron ions by UV-vis during centrifugation and storage. ${ }^{16}$ Results of the MTT assays for K562 cells exposed to all SPION samples are shown in Figure 6a. Average results are illustrated in Figures 6b,c for K562 and L929 cells, respectively.

All synthesized SPION samples demonstrated acceptable levels of cell viability following exposure, with none demonstrating toxic effects at the concentrations tested. In addition to the effects of exposure time and concentration, reductions in cell viability depended on the physical characteristics of the SPION. Different shapes and sizes, which are affected by the composition and reaction conditions during formation, impart different effects to the cells. The SPION used in this study have a narrow size distribution and are dispersed in a PVA polymeric substrate (Figures 7a,c), providing good cell viability. Parts b and d of Figure 7 demonstrate the diffraction patterns of SPION, which show the spinel structure. For instance, Figure $7 \mathrm{~d}$ highlights the corresponding (400), (422), (440), (533), and (800) planes of $\mathrm{Fe}_{3} \mathrm{O}_{4}$. In addition, the lattice fringes are illustrated in Figure 7e. In contrast, samples containing singular SPION nanoparticles, such as $\mathrm{S}(9000) \mathrm{M}(1.6)$, had comparatively reduced cell viability due to their higher chemical reactivity (Figure 7f).

3.1.2. Biocompatibility of SPION-Flow Cytometery. The toxicity of SPION may relate to the ability of SPION to damage DNA via magnetite oxidation. Previously, on the basis of a Comet assay in A549 human lung epithelial cells, Karlsson et al. ${ }^{29}$ suggested that magnetite nanoparticles can cause low levels of DNA toxicity by oxidative effects. Few studies have 


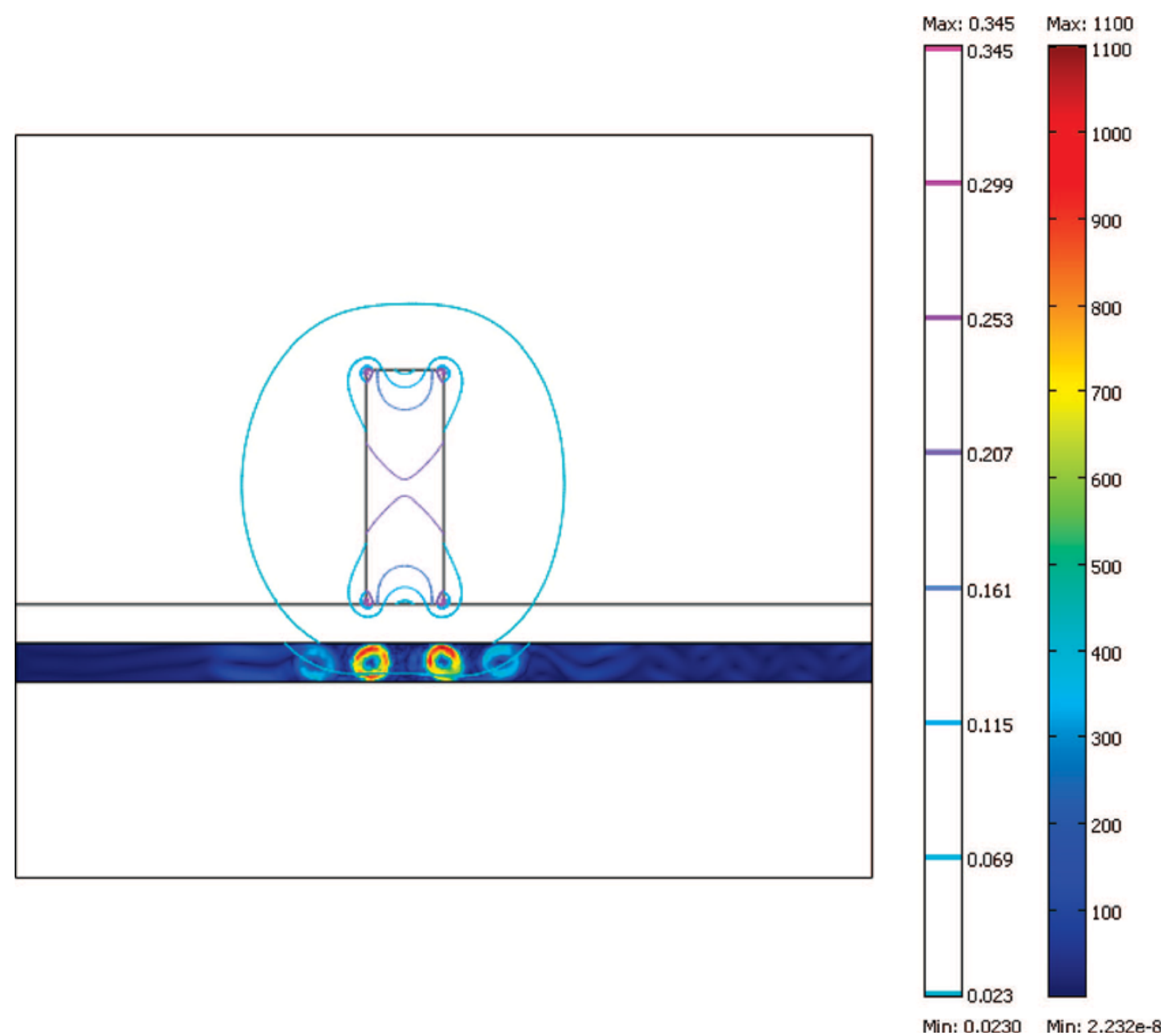

Figure 10. Contour lines of magnetic flux density and ferrofluid velocity surface contours in the blood vessel model (note: results are shown for $t=1 \mathrm{~s}$ for $\mathrm{S}(12600) \mathrm{M}(1.1)$ ). At the observation point marked in Figure 9, the value of magnetic flux density is $27.7 \mathrm{mT}$. Units of the values in the left and right legend are Tesla and $\mathrm{mm} / \mathrm{s}$, respectively.

demonstrated oxidative stress in relation to nanomaterials toxicity in both cell and fish models. ${ }^{30-33}$ Flow cytometry analysis indicates that the cells did not face apoptosis due to SPION exposure even at high molarities of $200 \mathrm{mM}$ (Figure 8). Since no apoptosis was detected with high SPION concentrations, it can be concluded that they are biocompatible for our target application dose.

3.2. Finite Element Model and Flow Simulation Results. Since we have shown that our PVA-coated nanoparticles are biocompatible, we wanted to investigate, by simulation, if we would be able to magnetically direct (stop/influence) them in large blood vessels. For this purpose, a 2D model triangular mesh was used, consisting of a blood vessel $1 \mathrm{~cm}$ in width, tissue $1 \mathrm{~cm}$ in width, an external magnet, and the surrounding environment (Figure 9); fluid flow is from left to right. The magnet is placed above the tissue to provide a permanent magnetic interaction with ferrofluids carried in the blood vessel. The fluid and magnetic boundary conditions are listed in Table 3. For this time-dependent analysis, the initial conditions are similarly listed in Table 3. The calculation process comprises two steps: the magnetic potential is initially computed, which is then used as an initial condition to calculate the fluid velocity field. The magnetic potential is calculated using a timeindependent stationary state to approximate the flux density distribution. The resulting values are then used to iteratively approximate the velocity field using the Navier-Stokes equations in the time domain. The fluid velocity and magnetic flux density contours are shown in Figure 10. The model confirms that the highest ferrofluid velocities occur as SPION pass through the region beneath the applied magnetic field. Simulations for all prepared SPION specimens were performed by varying the $\alpha$ and $\beta$ parameters (Figure 11).

To assess the impact of the magnetic field on fluid velocities, the velocity field was computed in the absence of the applied field. Since the inflow boundary is governed by a sinusoidal heart beat function, the resulting fluid velocity field was observed to be similarly sinusoidal. The peak fluid velocity without an external magnet was $358 \mathrm{~mm} / \mathrm{s}$, with no trace of turbulence detected. In contrast, application of the external magnetic field increased the peak velocity field to $835 \mathrm{~mm} / \mathrm{s}$, with the ferrofluid demonstrating turbulent flow. Some turbulence effects can be seen in Figure 10 near and under the external magnet. The chaotic nature of the flow is more closely realized in Figure 10, where each velocity component comprises a fluctuating trend. Between $t=0.5$ and $t=1 \mathrm{~s}$, during which time the (heart) inflow velocity vanishes (see also Figure 2), the magnet effect can be more dominant and the vertical velocity values are both positive and of a higher order of magnitude. Hence, during the diastole stage of a cardiac cycle the particles may collide with the tissue more effectively.

Although the size range of the modeled SPION was narrow $(3-5.5 \mathrm{~nm})$, different $x$ and $y$ fluid velocities were detected, even in a vessel as small as $1 \mathrm{~cm}$ (because of different hydrodynamic size). This indicates that the SPION synthesis parameters play an important role in ferrofluid behavior in vessels. Variations in the fluid velocity at a defined observation 
(a)
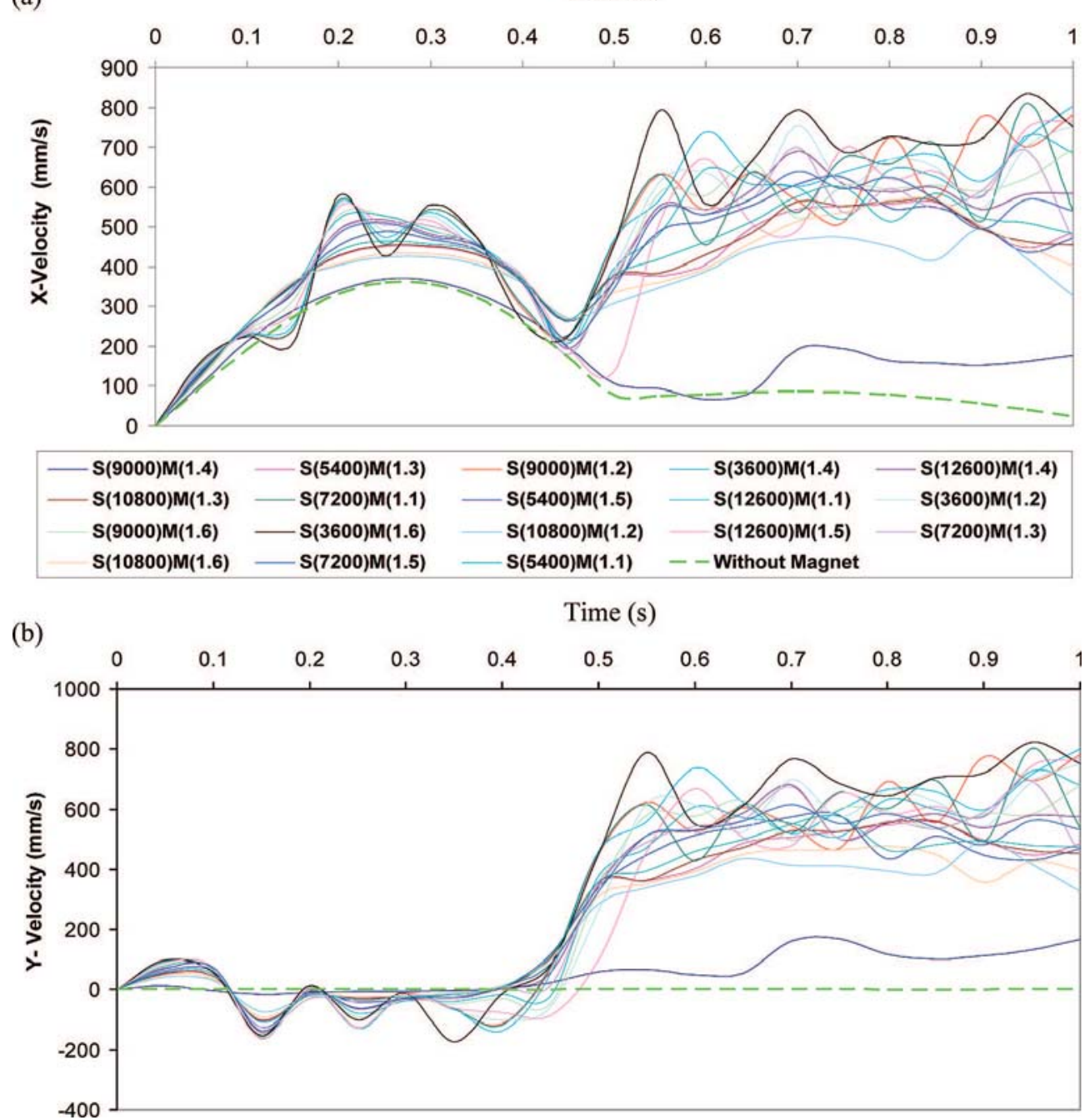

Figure 11. $x$ and $y$ velocities of the ferrofluid containing different nanoparticle samples. Results are presented for the observation point shown in Figure 8. Notice the increased fluctuations and peak velocities compared to the case without an external magnet. The SPION sample code is given in Figure 1.
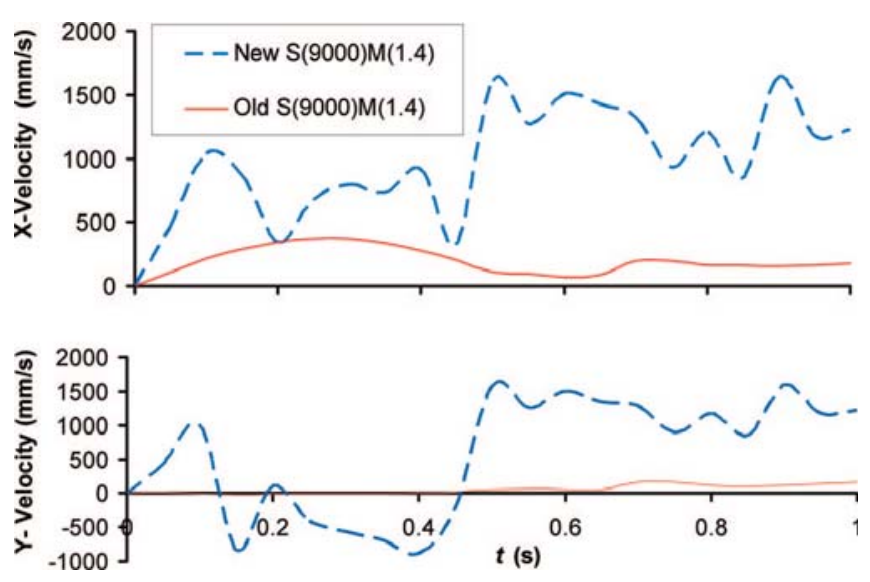

Figure 12. Comparison of the old (under magnet magnetization $=5$ $\times 10^{4}$ ) and new (under magnet magnetization $=3 \times 10^{5}$ ) $x$ and $y$ velocities of the ferrofluid containing the $\mathrm{S}(9000) \mathrm{M}(1.4)$ nanoparticle sample under an increased external magnetic field. The SPION sample code is given in Figure 1.

point in the vessel for different samples are shown in Figure 11. Notable changes were found in the $y$ velocity before and after $0.5 \mathrm{~s}$, which is related to flow pressure variation following sinusoidal heart beats. Interestingly, an interaction between the stirring rate $(S)$ and molarity $(M)$ was identified in the model.
For example, at a given molarity of 1.1, a comparison of peak velocities between $\mathrm{S}(12600) \mathrm{M}(1.1)$ and $\mathrm{S}(7200) \mathrm{M}(1.1)$ suggests that a higher stirring rate would result in a higher ferrofluid velocity amplitude. When the same comparison was made at a different constant molarity; e.g., between S(3600)M(1.6) and $\mathrm{S}(10800) \mathrm{M}(1.6)$, an opposite trend was observed. This means that the individual effect of a processing parameter on the velocity amplitude depends on the interaction of the preparation parameters. These results confirm our previous observation of interactions between the synthesis parameters and magnetization of SPION. ${ }^{16}$ Another noteworthy observation from Figure 11 is that for samples with low magnetic properties such as S(9000)M(1.4) (compare to Figure 9), the resulting velocity field is also of a low magnitude.

Finally, to investigate the effect of magnetic field strength, the applied field was increased by a factor of 10. The effect of increasing the field strength on the $x$ and $y$ velocities is shown in Figure 12. Under the increased field, the velocity of the ferrofluid along the vessel ( $x$ velocity) is increased by about 5 times, whereas the velocity component normal to the tissue $(y$ velocity) has amplified by about 10 times. While it remains to be verified, it is believed that to facilitate a drug delivery process, higher $y$ velocities would cause particles to collide more strongly and frequently with the vessel walls, thereby increasing the 
chance of attachment. The simulation results in Figure 12 suggest that higher external magnetic fields can increase the colliding velocities, but at the same time they may result in higher velocity fluctuations. A future multiobjective optimization study may be worthwhile to find conditions under which an optimal colliding velocity and direction with a minimal fluctuation may be realized.

\section{Conclusion}

The nanoparticles considered in this work were SPION composed of a magnetite core and a PVA coating. An MTT assay was used to investigate the biocompatibility of SPION of various compositions using L929 and K562 cells. All compositions tested demonstrated acceptable levels of cell viability following exposures of up to $20 \mathrm{mM}$ iron concentration for up to $48 \mathrm{~h}$. Flow cytometry tests and microscopic investigations showed neither apoptosis nor necrosis took place in cells exposed to SPION. A multiphysics finite element model was also developed to study the effects of an applied magnetic field on SPION in a simulated blood vessel. The FEM model iteratively solved coupled Maxwell and Navier-Stokes equations to predict both the induced magnetic flux density and fluid velocity fields. Simulation results suggest that both the strength of the applied magnetic field and the magnetic properties of SPION affect the velocity field fluctuations (flow turbulence) and amplitude (peak velocity). In turn, the magnetic properties are related to the processing parameters, namely, the stirring rate and $\mathrm{NaOH}$ molarity. These parameters showed some interactions in defining the shape and amplitude of the velocity field. Similar interactions were previously seen for optimizing the shape and size of the nanoparticles. Finally, it was noted that a more turbulent flow forms under an increased external field. A velocity field with less fluctuation and higher amplitude, especially in the direction normal to tissue, is believed to be preferable to facilitate drug delivery.

Acknowledgment. The valuable comments by Mr. P. Sasanpour from Sharif University of Technology is highly appreciated.

Supporting Information Available: Full experimental data. This material is available free of charge via the Internet at http:// pubs.acs.org.

\section{References and Notes}

(1) Mahmoudi, M.; Simchi, A.; Milani, A. S.; Stroeve, P. Proc. 2nd Conf. Nanostruct. (Kish Island, Iran) 2008, 66-68.

(2) Mornet, S.; Vasseur, S.; Grasset, F.; Duguet, E. J. Mater. Chem. 2004, 14, 2161-2175. 1997.
(4) Corr, S. A.; Rakovich, Y. P.; Gun'ko, Y. K. Nanoscale Res. Lett. 2008, 3, 87-104.

(5) Zaitsev, V. S.; Filimonov, D. S.; Presnyakov, I. A.; Gambino, R. J.; Chu, B. J. Colloid Interface Sci. 1999, 212, 49-57.

(6) Kang, Y. S.; Risbud, S.; Rabolt, J. F.; Stroeve, P. J. Chem. Mater. 1996, 8 (9), 2209-2211. 198.

(7) Berry, C. C.; Curtis, A. S. G. J. Phys. D: Appl. Phys. 2003, 36,

(8) Moghimi, S. M.; Hunter, A. C.; Murray, J. C. Pharm. Rev. 2001, 53,283

(9) Gupta, A. K.; Gupta, M. Biomaterials 2005, 26, 3995-4021.

(10) Harris, J. M.; Martin, N. E.; Modi, M. Clin. Pharmacokinet. 2001, $40,539-551$

(11) Xue, B.; Sun, Y. J. Chromatogr. A 2001, 921, 109-119.

(12) Chen, H.; Kaminski, M. D.; Ebners, A. D.; Ritter, J. A.; Rosengart, A. J. Proceedings of the 3rd Annual International IEEE EMBS Special Topic Conference on Microtechnologies in Medicine and Biology, Kahuku, Oahu, HI; 2005.

(13) Xie, Y.; Kaminski, M. D.; Mertz, C. J.; Finck, M. R.; Guy, S. G.; Chen, H.; Rosengart, A. J. Proceedings of the 3rd Annual International IEEE EMBS Special Topic Conference on Microtechnologies in Medicine and Biology, Kahuku, Oahu, HI; 2005; 12-15.

(14) Saltzman, W. M. Oxford University Press: Oxford, U.K., 2001.

(15) Haddish-Berhane, N.; Nyquist, C.; Haghighi, K.; Corvalan, C.; Keshavarzian, A.; Campanella, O.; Rickus, J.; Farhadi, A. J. Contr. Rel. 2006, 110, 314-322.

(16) Mahmoudi, M.; Simchi, A.; Imani, M.; Milani, A. S.; Stroeve, P. J. Phys. Chem. B 2008, 112, 14470-14481.

(17) Gupta, A. K.; Curtis, A. S. G. Biomaterials 2004, 25, 3029-3040.

(18) Petri-Fink, A.; Chastellain, M.; Juillerat-Jeanneret, L.; Ferrari, A.; Hofmann, H. Biomaterials 2005, 26, 2685-2694.

(19) Pieters, R.; Huismans, D. R. Br. J. Cancer 1989, 59, 217-220.

(20) Bolton, S. 2nd ed.; Dekker: New York., 1990.

(21) Saleh, O. A.; Blalock, W. L.; Burrows, C.; Steelman, L. S.; Doshi, P. D.; McKearn, J. P.; McCubrey, J. A. Int. J. Mol. Med. 2002, 10 (4), 385-394.

(22) Reinhold, W. C.; Kouros-Mehr, H.; Kohn, K. W.; Maunakea, A. K.; Lababidi, S.; Roschke, A.; Stover, K.; Alexander, J.; Pantazis, P.; Miller, L.; Liu, E.; Kirsch, I. R.; Urasaki, Y.; Pommier, Y.; Weinstein, J. N. Cancer Res. 2003, 63 (5), 1000-1011.

(23) Lohakan, M.; Junchaichanakun, P.; Boonsang, S.; Pintavirooj, C. IEEE Conf. Ind. Electron. Appl. (2nd) 2007, 231-234.

(24) Chakrabarty, S. P.; Hanson, F. B. Proceedings of the 44th IEEE Conference on Decision and Control, and the European Control Conference, Seville, Spain; 2005.

(25) Robert, W. F.; Alan, T. M.; Philip, J. P. John Wiley \& Sons: New York, 2004; pp 187-215.

(26) Trenado, C.; Strauss, D. J. Math. Model. Biol. Syst. 2007, 275280, ISBN 978-0-8176-4557-1.

(27) Milani, A. S.; Nemes, J. A. ASME J. Eng. Mater. Technol. 2004, 126 (4), 443-449. 82.

(28) Häfeli, U. O.; Pauer, G. J. J. Magn. Magn. Mater. 1999, 194, 76-

(29) Karlsson, H. L.; Cronholm, P.; Gustafsson, J.; Moller, L. Chem. Res. Toxicol. 2008, 21, 1726-1732.

(30) Hussain, S. M.; Hess, K. L.; Gearhart, J. M.; Geiss, K. T.; Schlager, J. J Toxicol. Vitro 2005, 19 (7), 975-983.

(31) Syes, C. M.; Gobin, A. M.; Ausman, K. D.; Mendez, J.; West, J. L.; Colvin, V. L. Biomaterials 2005, 26, 7587-7595.

(32) Gurr, J. R.; Wang, A. S.; Chen, C. H.; Jan, K. Y. Toxicology 2004, $213,66-73$.

(33) Oberdorster, E. Health Perspect. 2004, 112, 1058-1062. 